\title{
Blended intelligence of FCA with FLC for knowledge representation from clustered data in medical analysis
}

\author{
Ch. Neelima ${ }^{1}$, S.S.V.N. Sarma ${ }^{2}$ \\ ${ }^{1}$ Department of Computer Science, Kakatiya University, Warangal, India \\ ${ }^{2}$ Department of Computer Science \& Engineering, Vaagdevi College of Engineering, India
}

\begin{tabular}{l}
\hline \hline Article Info \\
\hline Article history: \\
Received Mar 19, 2018 \\
Revised Jul 6, 2018 \\
Accepted Jul 28, 2018 \\
\hline
\end{tabular}

Keyword:

Formal concept analysis (FCA) Formal implicated concept (FICpt) Fuzzy inference rules (FIR) Description logics (DL) Fuzzy logic controller (FLC) Formal implicated context (FICtx)

Fuzzy logic implicated DL (FLiDL)

Fuzzy logic implicated FCA (FLiFCA)

\begin{abstract}
Formal concept analysis is the process of data analysis mechanism with emergent attractiveness across various fields such as data mining, robotics, medical, big data and so on. FCA is helpful to generate the new learning ontology based techniques. In medical field, some growing kids are facing the problem of representing their knowledge from their gathered prior data which is in the form of unordered and insufficient clustered data which is not supporting them to take the right decision on right time for solving the uncertainty based questionnaires. In the approach of decision theory, many mathematical replicas such as probability-allocation, crisp set, and fuzzy based set theory were designed to deals with knowledge representation based difficulties along with their characteristic. This paper is proposing new ideological blended approach of FCA with FLC and described with major objectives: primarily the FCA analyzes the data based on relationships between the set of objects of prior-attributes and the set of attributes based prior-data, which the data is framed with data-units implicated composition which are formal statements of idea of human thinking with conversion of significant intelligible explanation. Suitable rules are generated to explore the relationship among the attributes and used the formal concept analysis from these suitable rules to explore better knowledge and most important factors affecting the decision making. Secondly how the FLC derive the fuzzification, rule-construction and defuzzification methods implicated for representing the accurate knowledge for uncertainty based questionnaires. Here the FCA is projected to expand the FCA based conception with help of the objective based item set notions considered as the target which is implicated with the expanded cardinalities along with its weights which is associated through the fuzzy based inference decision rules. This approach is more helpful for medical experts for knowing the range of patient's memory deficiency also for people whose are facing knowledge explorer deficiency.
\end{abstract}

Copyright (C) 2019 Institute of Advanced Engineering and Science. All rights reserved.

\section{Corresponding Author:}

Ch. Neelima,

Department of Computer Science,

Kakatiya University,

Hanamkonda, Warangal, India.

Email: neelima.chilagani@gmail.com

\section{INTRODUCTION}

Formal Concept Analysis (FCA) is a process with the data analysis feature which represents and obtains the embedded relationships between the objects based attributes and the objects based set of attributes. Formal concept analysis is the process of data analysis mechanism with emergent attractiveness across various fields such as data mining, robotics, medical, big data and so on. It helpful to analyzes the generalized data by deriving the relationship between an exacting set of objects and an exacting set of attributes of concern objects. In FCA, the data is represented with the help of structured implicated data-units 
by deriving the prescribed perception of concepts of human's brain thinking nature for generating the significant intelligible analysis statements and FCA is also holding another advantage of conceptual clustering technique which is used to offer the intended similes for the perceptions of abstracts along with the data-unit productivity implication [1]. Formal Concept Analysis (FCA), pioneered in the 1980's by Wille [2], is a method for extracting formal concepts-natural clusters of objects and attributes-from binary objectattribute relational data. FCA has great appeal in the context of knowledge discovery [3], information retrieval [4] and social networking analysis applications [5] because arranging data as a concept lattice yields a powerful and intuitive representation of the dataset [4], [6]. The FCA based formal context is derives the set of objects of objectives and attributes of objects through the binary relationship mechanism, but in real-time scenario it is supplementary significant to judge the straight relationships between objects of individual objectives of concern data-sets [7]. The definitional arguments stay unsettled, mainly because the psychologists and anthropologists are acknowledged through the various languages with help of logical symbol based structure which is formed by the individuals to signal meanings suggest various oncologists [9].

In various fields of applications has faced difficulties while processing the indistinguishable formless knowledge which is not suitable to robust into the classical logics of binary innovation mechanism. The binary based implicated representations such as the combinational answers of yes or no, true or false and 1 or 0 is difficult to generate the significant answers for the some complex questions such as whether a place is large or whether two states are close to each other [10]. The various range of size of degrees and proximity computations are used to represent the vague knowledge. Fuzzy based logical implications are best fit for deriving the scale of truth degree values to represent the vague knowledge. From these advancements, firstly it has formalized for propositional based implicated logics [11] and it has computationally implicated with various other logics along with their associated logical formalisms sequences such as the FCA and description logics (DL) [12], [13]. While implementing the fuzzy logic sequences to a past designed formalism some complications are noticed such as whether the real data-unit intermission values will be used for the set of degrees of truth values or used for the set of a supplementary degrees of truth values of complex lattices, retrieval of conjunction based semantics, In existing proposed methodology to decide which part of functionalities are continued for furthered execution without any modifications and which logical are missing whether these laggings logics will successfully solvable by the fuzzy logic based matching part. There type of decisional based thinking's are generated by using the fuzzy logic implicated FCA (FLiFCA) and fuzzy logic implicated DL (FLiDL). Researchers has proposed various approaches by using the FCA implicated DL sequences. Some approaches are executed with help of the computational based concept sequences [14], some approaches [15] ontology based computational progression which is used for developing and retrieving the embedded based learning data units by taking the support of graph implicated data sets [16], [17]. For executing the conjunctions in excess of concept based data, the set of attributes (SoA) are used for representing the objects in FCA based approached developments and the concept based descriptions are used for representing the individuals in DL based methods. The same class of semantics are flexible, sharable for executing the SoA in FCA and concept names based conjunctives in DL based methods [16].

FCA is helpful to generate the new learning ontology based techniques. The major problems are identified in medical field, some growing kids are facing the problem of representing their knowledge from their gathered prior data which is in the form of unordered and insufficient clustered data which is not supporting them to take the right decision on right time for solving the uncertainty based questionnaires. the FCA is projected to expand the FCA based conception with help of the objective based item set notions considered as the target which is implicated with the expanded cardinalities along with its weights which is associated through the fuzzy based inference decision rules. This approach is more helpful for medical experts for knowing the range of patient's memory deficiency also for people whose are facing knowledge explorer deficiency.

\section{PAPER OBJECTIVES}

This paper is proposing new ideological blended approach of FCA with FLC and described with major objectives: The FCA analyzes the data based on relationships between the set of objects of priorattributes and the set of attributes based prior-data, which the data is framed with data-units implicated composition which are formal statements of idea of human thinking with conversion of significant intelligible explanation. How the FLC derive the fuzzification, rule-construction and defuzzification methods implicated for representing the accurate knowledge for uncertainty based questionnaires. This approach is more helpful for medical experts for knowing the range of patient's memory deficiency also for people whose are facing knowledge explorer deficiency. 


\section{FCA BASED IMPLICATIONS}

The FCA implications will derive two major objective based classifications such as formal implicated contexts (FICtx) and formal concepts (FICpt) as shown in Figure 1.

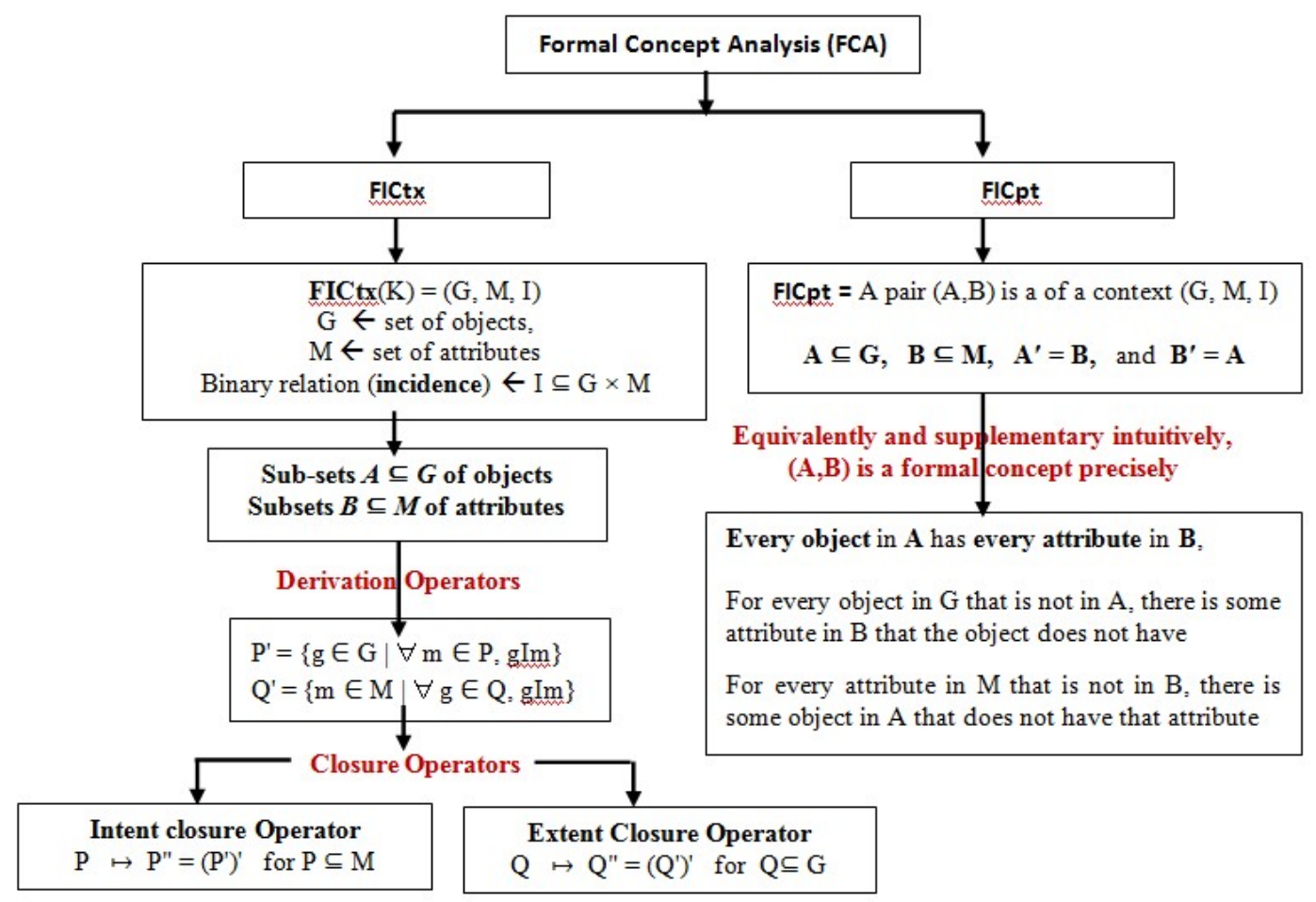

Figure 1. Execution and computational sequences of FCA based formal implicated contexts (FICtx) and formal concepts (FICpt)

\section{FUZZY LOGIC IMPLICATIONS}

The classical based set ( $\mathrm{CbS}$ ) theory and fuzzy based set (FbS) theory are the major basic computational components for designing and executing the embedded based systems. The CbS theory is representing the sets with an objects either it is member or not and the FbS theory is deriving the classes of degree for representing the membership functions, the membership function based object degrees are represented by real numbers such as 0 treated as no value in membership and 1 treated as full membership or some times between 0 and 1 [8]. FLC based reasoning (FLCbR) along with the graded concepts is holding the various advantages along with the various practical applications based uses [18]. FLCbR is extensively using in robotics, home need appliances, pattern recognition, image processing and many more. The FLCbR is best suitable in soft computing based hybrid systems such as fuzzy based neural system, fuzzy implicated genetic algorithms, neural-genetic systems, virtual and artificial intelligence and their application sequences [19]. The FLC system implicated microcontroller exists two division which is connected with the object code based correlated sectors. The first division deriving the feasibility to perform the functionalities of FLC based algorithm such as fuzzification, inference rule based assessment and defuzzification along with the FLC inference rule based mechanism [10]. Another division is help full to prepared the FLC based inputs and output come response along with application depended specific inference rules to carry towards to execute the FLC rule based mechanism. In FLC system, the Granulation is represented as in form of relations of input or output variables and in the form of information compression of variables.

\section{METHODOLOGY IMPLICATIONS}

In medical field some growing kids are facing the problem of representing their knowledge from their gathered prior data which is in the form of unordered and insufficient clustered data which is not supporting them to take the right decision on right time for solving the uncertainty based questionnaires. The medical assumptions based process deviate in the degree parameters which involves attempting to treaty with different cause difficulties aspect of hypothesis such as importance of relative-objectives, varied-postulation 
and the measuring the weak and strong relationships between questionnaires along with expected results which is gathered from their clients memory cells. In this process the FCA data analysis and FLC concept based lattices are more useful to retrieve the related knowledge from their client with this represented knowledge doctors will get the conclusion about their mental level of knowledge retrieval and is help full to prescribe the concern medicine to improve their reasoning skills. The FCA is used to analyze the data and FLC concept based lattices based inference rules used to perform the decision making methods with the help of the concern FLC reasoning approach for knowing the knowledge representation level or range of the children or clients and for deriving some class of conceptualization for the medical field by analyzing the medical test case based texts related to the generating the knowledge based on the some questionnaires. The table1 is representing the derived formal context based objects along with the concern attributes with help of related text-fields. Here the assumptions are build by concerning the relations which is represented in Table 1 , they are holding the additional and-or insufficient in their intelligence that we have very closer in day to day life activities and all non-occurring relations in the text are considered as partial negative opinions. Here the classes are generated with help of group of objects and generating the concept based chain of relation by analyzing their concern shared linguistic-contexts of objects. Here the meaningful classes are formed along with their hierarchies by using the cluster techniques based assumptions used for generating the group terms for knowledge retrieval process based on their age group. Table 1 is representing the concept lattice of the FICtx values illustrate in Figure 2 which is representing the relationships between the various nodes of objects along with their concern attributes and also it altered into a biased-order in a simple way of the removable mode of bottom object attribute by establishing an ontological concept for every node of FICpt along with their names by representing the concern intent and it is initiated with the sub-concept for every object based attribute in the dependent of the FICpt in concern questionnaires generated by the expert.

The natural language parser is helpful for deriving the essential object based dependencies from the words of concern attributes and every answer based statements are generated with help of FLC concept based lattices intent (FLCCLI) which is representing the language based depiction, FLC concept based lattices extent (FLCCLE) which is representing the SoO of concern objectives used for referring the depiction, FLC concept based lattices context (FLCCLC) in the concern words and these dependencies achieved through the in a simple way of execution helpful to retrieve the range of concern knowledge from the clients and to make possible by the human sympathetic of mechanically derived lattices and concept hierarchies with concern objects along with their attributes. This assumption of entirety of information is helpful to find all the possible occurrences by comparing the attributes of objects of individual with some group of attributes of objects by conserving the combination of SoO and SoA for generating the FCA implicated fuzzy sets which is needed to retrieve the knowledge based order and choices of the questionnaires as mentioned in Table 2 which is representing the logical attributes: fuzzy attributes with concern FCA implicated FLC lattice based computations such as Gödel based t-norm, intuition, hedge and various range of fuzzy sets as shown in Figure 3 and in section 5.2. In the experimental results generated the context with 8 number of objects, 9 number of attributes, 17 number of nodes and 27 number of arcs of lattice based relationship. Table 1 shows a comparison of various optimization techniques based on certain criteria

Table 1. Logical attributes with crisp attributes and objects with concern relationships in FICtx

\begin{tabular}{|c|c|c|c|c|c|c|c|c|c|}
\hline & Road & Water & Air & Wheels & Wings & $\begin{array}{c}\text { Passenger } \\
\text { Transportion }\end{array}$ & $\begin{array}{c}\text { Goods } \\
\text { Transporation }\end{array}$ & $\begin{array}{c}\text { Fuel } \\
\text { Usage }\end{array}$ & $\begin{array}{c}\text { Animal } \\
\text { Usage }\end{array}$ \\
\hline Cars & $\mathrm{X}$ & & & $\mathrm{X}$ & & $\mathrm{X}$ & $\mathrm{X}$ & $\mathrm{X}$ & \\
\hline Bikes & $\mathrm{X}$ & & & $\mathrm{X}$ & & & & $\mathrm{X}$ & \\
\hline Flights & & & $\mathrm{X}$ & $\mathrm{X}$ & X & $\mathrm{X}$ & $\mathrm{X}$ & $\mathrm{X}$ & \\
\hline Ships & & $\mathrm{X}$ & & & & $\mathrm{X}$ & $\mathrm{X}$ & $\mathrm{X}$ & \\
\hline Helicopter & & & $X$ & & & $\mathrm{X}$ & & $\mathrm{X}$ & \\
\hline Auto & $\mathrm{X}$ & & & $\mathrm{X}$ & & $\mathrm{X}$ & $\mathrm{X}$ & $\mathrm{X}$ & \\
\hline Bicycle & $\mathrm{X}$ & & & $\mathrm{X}$ & & & & & \\
\hline Bullacord & & & & $\mathrm{X}$ & & $\mathrm{X}$ & & & $\mathrm{X}$ \\
\hline
\end{tabular}

\subsection{FLC concept based lattices}

FLC concept based lattices are best for solving the complex problems in big-data, soft computing and robotics based investigative analysis process. The FLC concept based lattices are majorly deriving the three components such as: FLC concept based lattices intent (FLCCLI) which is representing the language based depiction, FLC concept based lattices extent (FLCCLE) which isrepresenting the SoO of concern objectives used for referring the depiction, FLC concept based lattices context (FLCCLC) which is represented with the concern universe of entire probable objects and the universe of entire probable attributes of objects along with the their related logical classification of the concern relation-type which the object 
possesses an attribute values then it indicates the relationships of SoA along with the SoA which is used to execute the functionalities for further development of the system. In FLCCLC, with help of substance of degree the decision making process can be occur such as whether an concern object is belongs to a concern concept or not and whether it consists an attribute or not, some time the decision is treated as crisp if the many attributes are holding the values in fuzzification [20]. In this approach the computational complexities are performed by the numerical values. Here the numerical values are allocated to every attribute by implicating with concern scaling factor. Finally the outcome responses are generated with help of table formation by deriving the related associates of every allocated object based values within the mentioned series to the numerical value by signifying the applicability of specified degree values. Here the FLCCLC are represented with the graphs with help of lattices and concern nodes along with their various diverse interconnectivity ranges [23]. The crisp based data implementation way is differ in FLC based DL and FLC implicated FCA. In generalized fuzzy logic sequences, sometimes the computational scenarios are not flexible for executing the operations, but in FLC implicated FCA is deriving the future of fuzzy based SoA [10]. The generalized DL based concept descriptions are similarly implementing the FLC based DL, here the only semantics are fuzzy based but they are not pure fuzzy logic. The Gödel represented t-norm is used accurately in the section of FLC based FCA where the semantics will partly covers by the fuzzy based DL which is most probably consent to synergies as in the crisp case of scenarios [24]. In Gödel based t-norm representations, sometimes it is holding the essential limit when the FLC based FCA implementation uses the weak-conjunction (WC) for the semantics of SoA while DL utilized the strong-conjunction (SC) for its concern semantics [25][26]. The SC and WC correspond solitary for the Gödel based t-norm. There is another limit in DL point of view which is the Gödel based t-norm is the solitary t-norm for which the standard DL based reasoning operations are acknowledged to be decisional scenario [21][22].

\subsection{FCA implicated fuzzy logic set, t-norms and hedges}

FLC is deriving the well-defined semantics for indicating the indistinct-data by using the scale of truth degrees of range values [27][28]. This approach representing the truth degrees interval values such as $[0.0,1.1]$ from the real-unit-interval $[0,1]$. FLC is deriving the various computational operational sequences to define the concern its semantics.

Table 2. Logical attributes along with the fuzzy attributes of input values

\begin{tabular}{|c|c|c|c|c|c|c|c|c|c|}
\hline & Road & Water & Air & Wheels & Wings & $\begin{array}{c}\text { Passenger } \\
\text { Transportion }\end{array}$ & $\begin{array}{c}\text { Goods } \\
\text { Transporation } \\
\end{array}$ & $\begin{array}{c}\text { Fuel } \\
\text { Usage }\end{array}$ & $\begin{array}{c}\text { Animal } \\
\text { Usage }\end{array}$ \\
\hline Cars & 1 & & & 0.9 & & 0.8 & 0.7 & 0.6 & \\
\hline Bikes & 1 & & & 0.9 & & & & 0.8 & \\
\hline Flights & & & 1 & 0.9 & 0.8 & 0.7 & 0.6 & 0.5 & \\
\hline Ships & & 1 & & & & 0.9 & 0.8 & 0.7 & \\
\hline Helicopter & & & 1 & & & 0.9 & & 0.8 & \\
\hline Auto & 1 & & & 0.9 & & 0.8 & 0.7 & 0.6 & \\
\hline Bicycle & 1 & & & 0.9 & & & & & \\
\hline Bullacord & & & & 1 & & 0.8 & & & 0.7 \\
\hline
\end{tabular}

\subsubsection{Godel based t-norm}

It represents the binary operation along with binary operator $U:[0,1] \times[0,1] \rightarrow[0,1]$ which is commutative, associative, monotone rule passing scenarios and finally it derives 1 as its outcome unit value. Here each incessant t-norm presents to mount to a binary based operator $\mathrm{V}:[0,1] \mathrm{x}[0,1] \rightarrow[0,1]$ which is derives the unique-operator fulfilling for all $\mathrm{z} \in[0,1]$.

$$
\mathrm{z} \leq \mathrm{x}=>\mathrm{y} \text { if and only if } \mathrm{x} U \mathrm{z} \leq \mathrm{y}
$$

\subsubsection{FCA based intuition}

The intuition is that the t-norm and the residuum can be used to interpret conjunction and implication, respectively. Among the many continuous t-norms perhaps the simplest one, and the one we shall be interested in, is the Gödel t-norm.

$$
\begin{array}{ll}
\mathrm{x} U \mathrm{y}=\min \{\mathrm{x}, \mathrm{y}\} & \\
\text { Residuum: } & \text { if } \mathrm{x} \leq \mathrm{y} \\
& \text { Then } \mathrm{x}=>\mathrm{y}=1 \\
& \text { Otherwise } \mathrm{y}
\end{array}
$$




\subsubsection{FCA based hedge}

It is a unary operator that is idempotent and satisfies $1^{*}=1, a^{*} \leq a$, and $(a=>b) * \leq a^{*}=>b^{*}$ for all $\mathrm{a}, \mathrm{b}[0,1]$. It is used for truth-stressing, to increase the contrast between 1 and the smaller truth values. A simple hedge is the globalization, defined as $1^{*}=1$ and $\mathrm{a}^{*}=0$ for $\mathrm{a} \neq 0$.

\subsubsection{FCA based fuzzy set}

Fuzzy sets are a central idea of Fuzzy Logics. Given a set $\mathrm{M}$ a fuzzy (sub-)set $\mathrm{T}$ of $\mathrm{M}$ is a function $\mathrm{T}: \mathrm{M} \rightarrow[0 ; 1]$, that maps each element of $\mathrm{M}$ to its membership degree in $\mathrm{T}$. The cardinality of a fuzzy set $\mathrm{T}$ is defined as the cardinality of its support $\{x \in M \mid T(x)>0\}$. Two fuzzy sets $T 1$ and T2 can be compared point wise by defining $\mathrm{T} 1 \subseteq \mathrm{T} 2$ iff $\mathrm{T} 1(\mathrm{x}) \leq \mathrm{T} 2(\mathrm{x})$ for all $\mathrm{x} \in \mathrm{M}$. alternatively, one can associate a subset-hood degree with $\mathrm{T} 1$ and $\mathrm{T} 2$ by defining;

$$
\mathrm{S}(\mathrm{T} 1, \mathrm{~T} 2)=\inf \mathrm{x} \in \mathrm{M} \mathrm{T} 1(\mathrm{x})=>\mathrm{T} 2(\mathrm{x})
$$

figure 2 shows the concept lattice of the FICtx values.

\begin{tabular}{|c|c|c|c|c|c|c|c|c|c|}
\hline & Road & Water & Air & Wheels & $\begin{array}{l}\text { Wings } \\
\text { S }\end{array}$ & $\begin{array}{l}\text { Passanger Tr } \\
\end{array}$ & Goods Transportatiox & Fuel Usage & Animal Usage $\bar{x}$ \\
\hline Cars $x$ & $\mathrm{x}$ & & & $\bar{x}$ & & $\mathrm{x}$ & $\bar{x}$ & $x$ & \\
\hline Bikes $x$ & $x$ & & & $\mathrm{x}$ & & & & $\mathrm{x}$ & \\
\hline Flights $x$ & & & $\mathrm{x}$ & $x$ & $x$ & $\mathrm{x}$ & $\mathrm{x}$ & $x$ & \\
\hline Ships $x$ & & $x$ & & & & $\bar{x}$ & $x$ & $x$ & \\
\hline Helicopter $x$ & & & $\bar{x}$ & & & $\bar{x}$ & & $x$ & \\
\hline Auto $x$ & $x$ & & & $x$ & & $\mathrm{x}$ & $\mathrm{x}$ & $x$ & \\
\hline Bicycle $x$ & $\mathrm{x}$ & & & $x$ & & & & & \\
\hline Bullacord $x$ & & & & $x$ & & $\mathrm{x}$ & & & $x$ \\
\hline
\end{tabular}

Figure 2. Experimented figure of logical attributes with crisp attributes and objects with concern relationships in FICtx

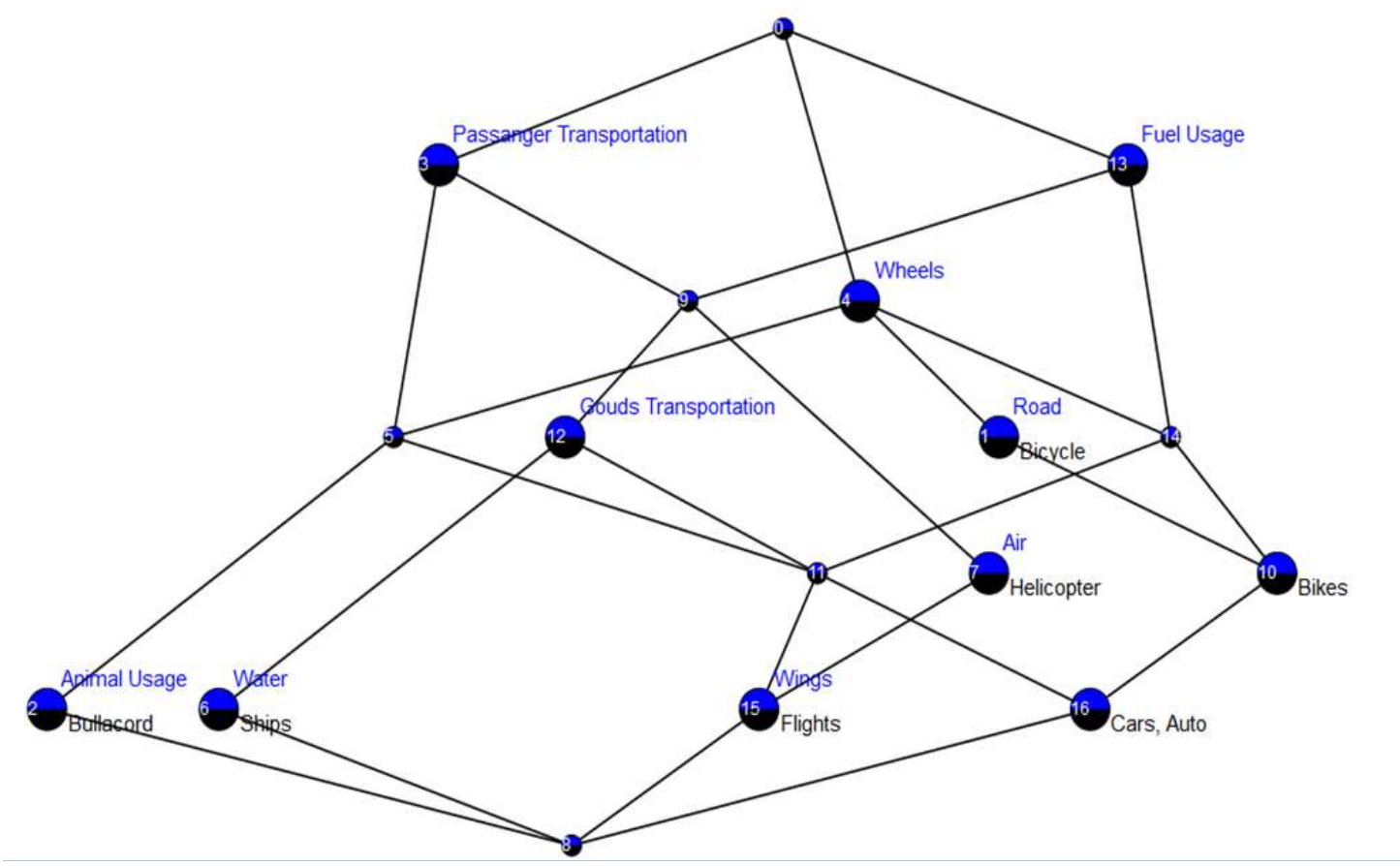

Figure 3. Nodes of lattices mechanically derived from relationship indication of vehicle representational knowledge based on Table 1 and Figure 2

\subsection{Crisp FCA implicated with fuzzy logic}

In the crisp setting has implicated with crisp FCA in addition to fuzzy FCA, as we shall need the crisp version of the Duquenne-Guigues Base in the later sections. 
In crisp FCA [11], data is typically represented in the form of cross tables such as the one in Table 1. More formally, a formal context is a triple $K=(G, M, I)$ where $G$ is a set, called the set of objects, $M$ is a set, called the set of attributes, and $\mathrm{I} \subseteq \mathrm{G} \times \mathrm{M}$ is a binary relation, called the incidence relation. For sets $\mathrm{A} \subseteq \mathrm{G}$ and $\mathrm{B} \subseteq \mathrm{M}$ the derivation operators are defined as

$$
\begin{aligned}
& \mathrm{A}^{\uparrow}=\{\mathrm{m} \in \mathrm{M} \mid \forall \mathrm{g} \in \mathrm{A}:(\mathrm{g}, \mathrm{m}) \in \mathrm{I}\} \\
& \mathrm{B}^{\downarrow}=\{\mathrm{g} \in \mathrm{G} \mid \forall \mathrm{m} \in \mathrm{B}:(\mathrm{g}, \mathrm{m}) \in \mathrm{I}\}
\end{aligned}
$$

The two derivation operators. ${ }^{\uparrow}$ and ${ }^{\downarrow}$ form an antitone Galois-connection. An implication $\mathrm{A} \rightarrow \mathrm{B}$, where $\mathrm{A}, \mathrm{B} \subseteq \mathrm{M}$, is said to hold in the context $\mathrm{K}$ if $\mathrm{A} \_\subseteq \mathrm{B} . \downarrow$.

A set of attributes $\mathrm{U} \subseteq \mathrm{M}$ respects $\mathrm{A} \rightarrow \mathrm{B}$ iffA does not $\subseteq$ of $\mathrm{U}$ or $\mathrm{B} \subseteq \mathrm{U}$. $\mathrm{A} \rightarrow \mathrm{B}$ follows from a set of implications $\mathrm{L}$ iff every set $\mathrm{U}$ that respects all implications from $\mathrm{L}$ also respects $\mathrm{A} \rightarrow \mathrm{B}$.

In a fuzzy context $K=(G, M, I)$ the incidence relation $I$ is a fuzzy relation, i.e. a fuzzy subset of $G x$ M. The derivation operators are defined for fuzzy subsets A of $G$ and fuzzy subsets B of M as follows:

$$
\begin{aligned}
& \mathrm{A}^{\uparrow}(\mathrm{m})=\inf \left(\mathrm{g}_{\in} \mathrm{G}\right)\left(\mathrm{A}(\mathrm{g})^{*}=>\mathrm{I}(\mathrm{g}, \mathrm{m})\right) \\
& \mathrm{B}^{\downarrow}(\mathrm{g})=\inf \left(\mathrm{m}_{\in} \mathrm{M}\right)(\mathrm{B}(\mathrm{m})=>\mathrm{I}(\mathrm{g}, \mathrm{m})
\end{aligned}
$$

In fuzzy FCA the implications are allowed to be fuzzy. A fuzzy implication is a pair written as $\mathrm{A} \rightarrow$ $B$ where $A$ and $B$ are fuzzy subsets of $M$. Let $U$ be a fuzzy subset of $M$. The degree to which $A \rightarrow B$ holds in $\mathrm{U}$ is defined as

$\left.\|\mathrm{A} \rightarrow \mathrm{B}\|_{\mathrm{U}}=\mathrm{S}(\mathrm{A}, \mathrm{U})^{*}\right)=>\mathrm{S}(\mathrm{B}, \mathrm{U})$

The degree to which $\mathrm{A} \rightarrow \mathrm{B}$ holds in IK is defined as

$\|\mathrm{A} \rightarrow \mathrm{B}\|_{\mathrm{IK}}=\min _{\mathrm{g} 2 \mathrm{G}}\|\mathrm{A} \rightarrow \mathrm{B}\|_{\mathrm{Ig}}$

Where Ig is the fuzzy set to which each $m \in M$ belongs with degree $I(g, m)$. Let $L$ be a fuzzy set of fuzzy implications. $\mathrm{A} \rightarrow \mathrm{B}$

A set $\mathrm{U} \subseteq \mathrm{M}$ is called a model of $\mathrm{L}$ if $\|\mathrm{A} \rightarrow \mathrm{B}\|_{\mathrm{U}} \geq \mathrm{I}(\mathrm{A} \rightarrow \mathrm{B})$ holds for every fuzzy Implication of

$\mathrm{A} \rightarrow \mathrm{B}$ follows from $\mathrm{L}$ to degree $\mathrm{q}$ if $\|\mathrm{A} \rightarrow \mathrm{B}\|_{\mathrm{U}} \geq \mathrm{q}$ for all models $\mathrm{U}$ of $\mathrm{L}$.

Figure 4 shows the visualized conceptual clusters related to vehicle representational knowledge compounds.

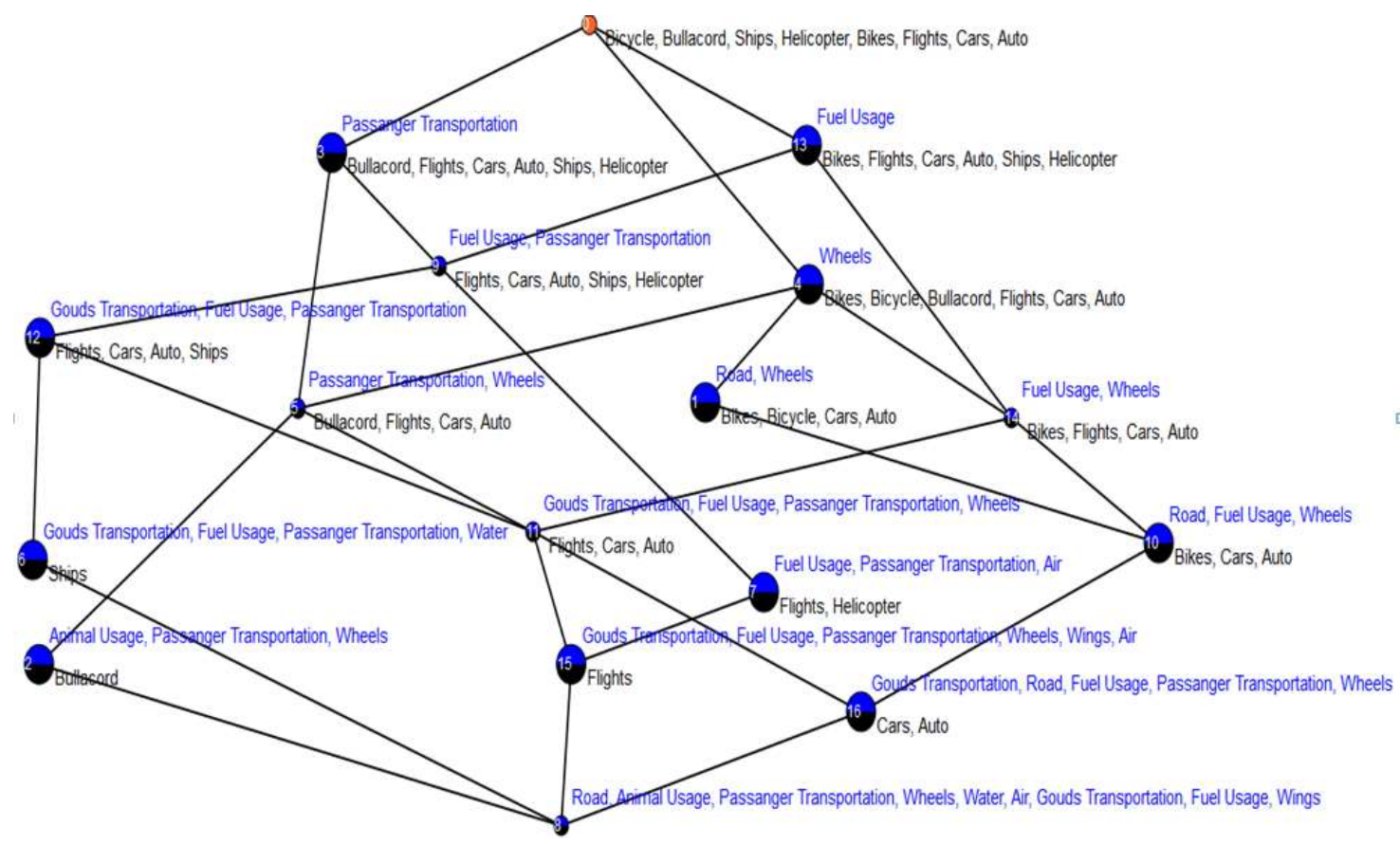

Figure 4. The resulting conceptual clustering of the text clusters along with visualized for the clusters related to vehicle representational knowledge compounds and generated the context with 8 objects, 9 attributes, 17 nodes and 27 arcs 
Figure 5 and 6 shows the logical attributes by concerning the partial input fuzzy attribute values and the complete fuzzy attribute values respectively

\begin{tabular}{|c|c|c|c|c|c|c|c|c|c|c|c|c|}
\hline & Road & Water & $x$ & Air & $\bar{x}$ & Wheels & Wings & $\mathrm{X}_{\text {Passanger Transpof }}$ & Goods Transport $\mathbf{x}$ & Fuel & $x$ & Animal Usage $x$ \\
\hline Cars $x$ & 1 & & & & & 0.9 & & 0.8 & 0.7 & 0.6 & & \\
\hline Bikes $x$ & 1 & & & & & 0.9 & & & & 0.8 & & \\
\hline Flights $\mathrm{X}$ & & & & 1 & & 0.9 & 0.8 & 0.7 & 0.6 & 0.5 & & \\
\hline Ships $X$ & & 1 & & & & & & 0.9 & 0.8 & 0.7 & & \\
\hline Helicopter $x$ & & & & 1 & & & & 0.9 & & 0.8 & & \\
\hline Auto $x$ & 1 & & & & & 0.9 & & 0.8 & 0.7 & 0.6 & & \\
\hline Bicycle $x$ & 1 & & & & & 0.9 & & & & & & \\
\hline Bullacord $x$ & & & & & & 1 & & 0.8 & & & & 0.7 \\
\hline
\end{tabular}

Figure 5. Logical attributes by concerning the partial input fuzzy attribute values

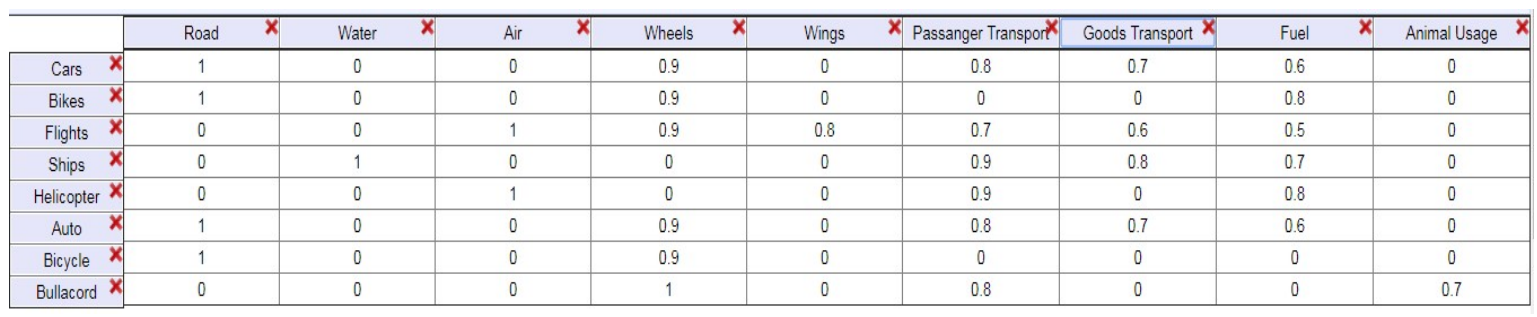

Figure 6. Logical attributes by concerning the complete fuzzy attribute values

Figure 7 shows the resulting of full concept lattice along with visualized clusters related to vehicle representational knowledge compounds and Figure 8 shows the graphical representation of fuzzy logic implicated interval relationship.

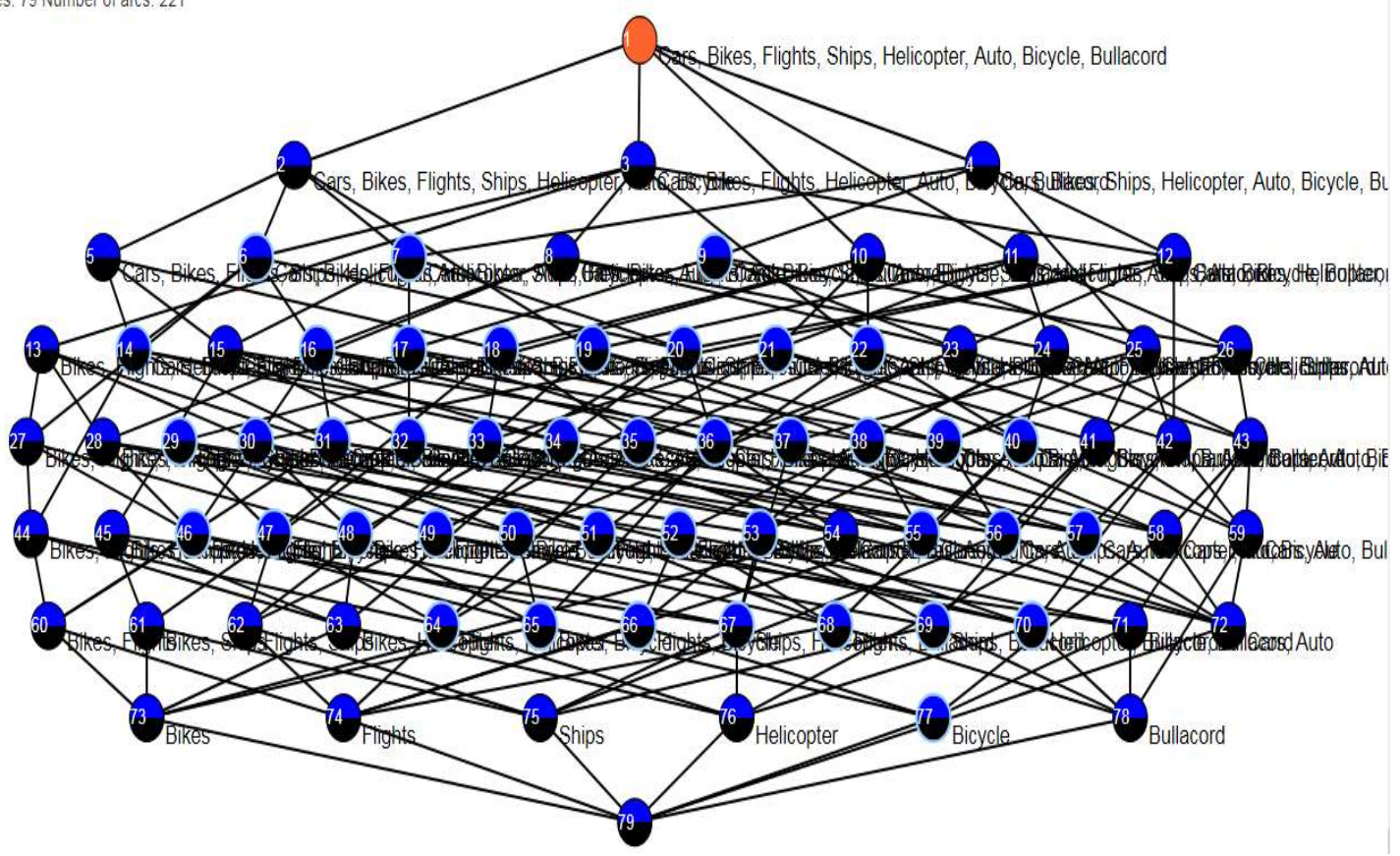

Figure 7. The resulting conceptual clustering of the text clusters along with visualized for the clusters related to vehicle representational knowledge compounds 


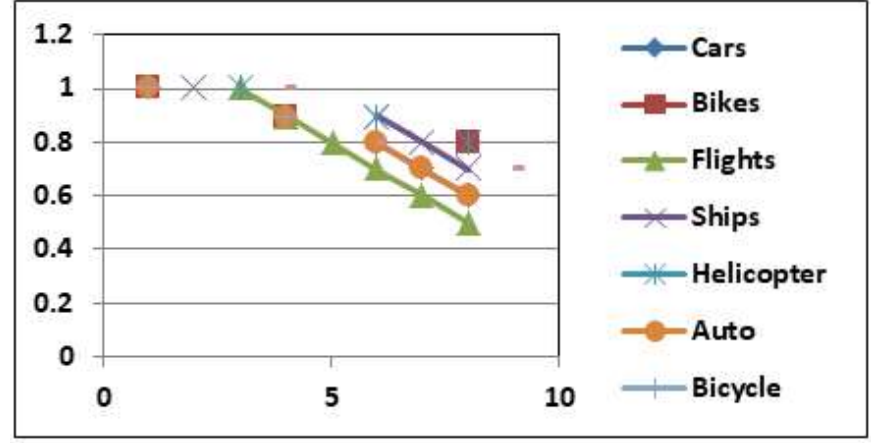

Figure 8. Graphical representation of fuzzy logic implicated interval relationships

Figure 9 shows the extents and intents along with logical values and Figure 10 shows a particular lattices of relationship of attributes of one object to another object.

\begin{tabular}{|l|}
\hline All Concepts of AOC poset: \\
\hline 1-Extent: Bikes, Bicycle, Cars, Auto, Intent : Road, Wheels \\
\hline 2-Extent: Bullacord, Intent : Animal Usage, Passanger Transportation, Wheels \\
\hline 3-Extent: Bullacord, Flights, Cars, Auto, Ships, Helicopter, Intent : Passenger Transportation \\
\hline 4-Extent: Bikes, Bicycle, Bullacord, Flights, Cars, Auto, Intent : Wheels \\
\hline 6-Extent: Ships, Intent : Goods Transportation, Fuel Usage, Passenger Transportation, Water \\
\hline 7-Extent: Flights, Helicopter, Intents : Fuel Usage, Passenger Transportation, Air \\
\hline 10-Extent: Bikes, Cars, Auto, Intents : Road, Fuel Usage, Wheels \\
\hline 12-Extent: Flights, Cars, Auto, Ships, Intent : Goods Transportation, Fuel Usage, Passenger \\
Transportation \\
\hline 13-Extent: Bikes, Flights, Cars, Auto, Ships, Helicopter, Intent : Fuel Usage \\
\hline 15-Extent: Flights,Intent : Goods Transportation, Fuel Usage, Passenger Transportation, Wheels, \\
Wings, Air \\
\hline 16-Extent: Cars, Auto, Intent : Goods Transportation, Fuel Usage, Passenger Transportation, Wheels \\
\hline
\end{tabular}

Figure 9. Output of multi valued multi-contexts of extents and intents along with the logical scale

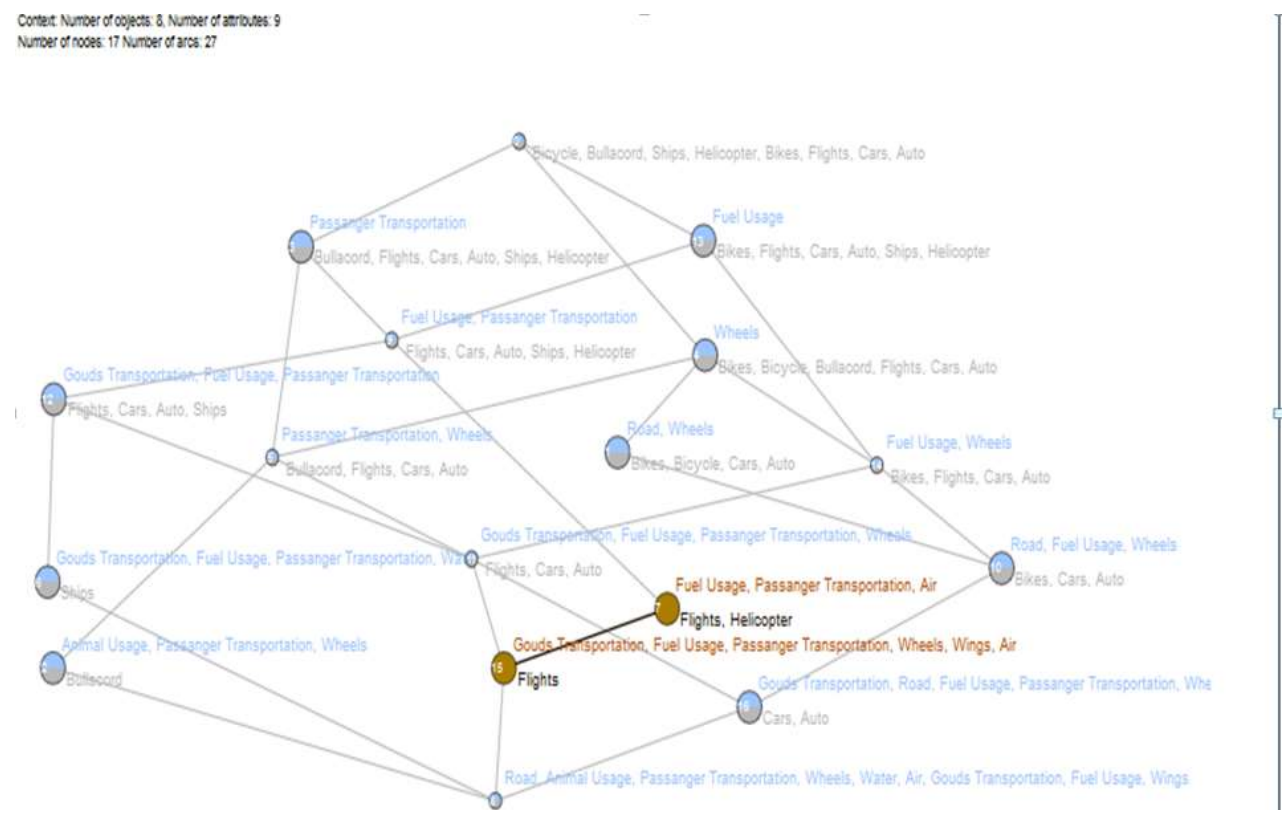

Figure 10.Particular lattices of relationship of attributes of one object to attributes of another object with the conceptual clustering mechanism 


\section{CONCLUSION}

FCA analyzes data which describe relationship between a particular set of objects and a particular set of attributes. The data are structured into units which are formal abstractions of concepts of human thought, allowing meaningful comprehensible interpretation. This paper is recommending new ideological blended approach of FCA with FLC and described with major objectives: primarily the FCA analyzes the data based on relationships between the set of objects of prior-attributes and the set of attributes based priordata, which the data is framed with data-units implicated composition which are formal statements of idea of human thinking with conversion of significant intelligible explanation. Suitable rules are generated to explore the relationship among the attributes and used the formal concept analysis from these suitable rules to explore better knowledge and most important factors affecting the decision making. Secondly how the FLC derive the fuzzification, rule-construction and defuzzification methods implicated for representing the accurate knowledge for uncertainty based questionnaires. This approach is more helpful for medical experts for knowing the range of patient's memory deficiency also for people whose are facing knowledge explorer deficiency.

\section{ACKNOWLEDGEMENTS}

I would like to thank to my supervisor S.S.V.N Sarma, my family, colleagues and friends.

\section{REFERENCES}

[1] https://www.cs.cmu.edu/afs/cs/project/jair/pub/volume24/cimiano05a-html/node3.html.

[2] Rudolf Wille., "Restructuring Lattice Theory: an Approach Based on Hierarchies of Concepts," In Ivan Rival, editor, Ordered sets, pages 445-470. Reidel, 1982.16 Xu. et al.

[3] Lotfi Lakhal, Gerd Stumme., "Efficient Mining of Association Rules Based on Formal Concept Analysis," In Formal Concept Analysis'05, pages 180-195, 2005.

[4] G'eraldine Polaillon, Marie-Aude Aufaure, B'en'edicte Le Grand, Michel Soto., "FCA for Contextual Semantic Navigation and Information Retrieval in HeterogeneousInformation Systems," In DEXA Workshops'07, pages 534539, 2007.

[5] V'aclav Sn'asel, Zdenek Horak, Jana Kocibova, Ajith Abraham., "Analyzing Social Networks Using FCA: Complexity Aspects," In Web Intelligence/IAT Work-shops'09, pages 38-41, 2009.

[6] Nathalie Caspard, Bernard Monjardet., "The Lattices of Closure Systems, Closure Operators, and Implicational Systems on a Finite Set: A Survey," Discrete Applied Mathematics, pages 241-269, 2003.

[7] Feng Jiang, Youxin Meng, Yun Liu, "Formal Concept Analysis In Relational Contexts," Granular Computing, 2008. GrC 2008. IEEE International Conference on, 31 October 2008, 10.1109/GRC.2008.4664730.

[8] Susan Haack., "Deviant Logic, Fuzzy Logic: Beyond The Formalism,".

[9] Alfred Korzybski., "Science and Sanity: An Introduction to Non-Aristotelian Systems and General Semantics," 5th ed., Forest Hills, N.Y.: Institute of General Semantics, 1995.

[10] Felix Distel., "Adapting Fuzzy Formal Concept Analysis for Fuzzy Description Logics," Institute of Theoretical Computer Science, Technische Universität Dresden, Dresden, Germany.

[11] Hájek, P., "Metamathematics of Fuzzy Logic (Trends in Logic)," Springer (2001).

[12] Belohlavek, R., "Fuzzy Relational Systems: Foundations and Principles," Kluwer (2002).

[13] García-Cerdaña, Á, Armengol, E, Esteva, F., "Fuzzy Description Logics And T-Norm Based Fuzzy Logics," Int. J. of Approx. Reasoning Vol. 51 pp. 632-655, 2010.

[14] Sertkaya, B., "Computing The Hierarchy Of Conjunctions Of Concept Names And Their Negations In A Description Logic Knowledge Base Using Formal Concept Analysis," In:Cont. to ICFCA '06, Dresden, Germany pp. 73-86, 2006.

[15] Baader, F, Ganter, B, Sattler, U, Sertkaya, B., "Completing Description Logic Knowledge Bases Using Formal Concept Analysis," In: IJCAI'07. 2007.

[16] Rudolph, S., "Exploring relational structures via FLE," In: Conceptual Structures at Work Springer. 233-233, 2004.

[17] Distel, F., "Learning Description Logic Knowledge Bases from Data Using Methods from Formal Concept Analysis," PhD thesis, TU Dresden, 2011.

[18] Kazuo Tanaka., "An Introduction to Fuzzy Logic for Practical Applications," Springer, 1996; Constantin Zopounidis, Panos M. Pardalos \& George Baourakis, Fuzzy Sets in Management, Economics and Marketing. Singapore; World Scientific Publishing Co. 2001.

[19] Stosberg, Mark (16 December 1996)., "The Role of Fuzziness in Artifical [sic] Intelligence," Minds and Machines. Archived from the original on 20 May 2013. Retrieved 19 April 2013.

[20] Radim Bělohlávek and Vilem Vychodil., "What is a fuzzy concept lattice?," Department of Computer Science, Palacky University, Olomouc, 2005.

[21] Bobillo, F., Delgado, M., Gómez-Romero, J., Straccia, U., "Fuzzy Description Logics Under Gödel Semantics, " Int. J. of Approx. Reasoning Vol. 50 No. 3; 494-514, 2009. 
[22] Li, et al., "Analysis and Simplification of Three-Dimensional Space Vector PWM for Three-Phase Four-Leg Inverters," IEEE Transactions on Industrial Electronics, vol. 58, pp. 450-464, Feb 2011.

[23] R. Arulmozhiyal and K. Baskaran, "Implementation of a Fuzzy PI Controller for Speed Control of Induction Motors Using FPGA," Journal of Power Electronics, vol. 10, pp. 65-71, 2010.

[24] Biao Xu, Ruair'1 de Fr'ein, Eric Robson, and M'iche'al 'O Foghl'u., "Distributed Formal Concept Analysis Algorithms Based on an Iterative MapReduce Framework," Telecommunications Software \& Systems Group, Waterford Institute of Technology, Ireland, arXiv:1210.2401v1 [cs.DC] 5 Oct 2012.

[25] Singh, P.K. and Aswani Kumar. Ch, "Bipolar Fuzzy Graph Representation Of Concept Lattice," Information Sciences, 288: 437-448, 2014

[26] Sarmah, A.K., Hazarika, S.M. and Sinha, S.K, "Formal Concept Analysis: Current Trends And Directions," Artificial Intelligence Review 44: 47-86, DOI:10.1007/s10462-013-9404-0, 2015.

[27] Dias, S.M. and Vieira, N.J., "Concept Lattices Reduction: Definition, Analysis And Classification," Expert Systems with Applications. Vol. 42 No. 20: 7084-7097, DOI: 10.1016/j.eswa.2015.04.044, 2015.

[28] Prem Kumar Singh , Cherukuri Aswani Kumar , Abdullah Gani, "A Comprehensive Survey On Formal Concept Analysis, Its Research Trends And Applications," International Journal of Applied Mathematics and Computer Science, Pp. 495-516, Feb 2016.

\section{BIOGRAPHIES OF AUTHORS}

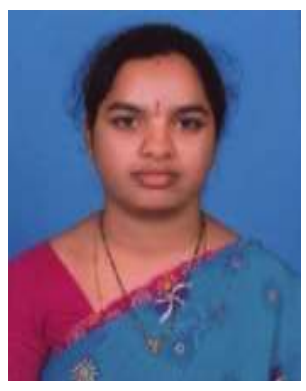

Working at Kakatiya University. Research interest are Computer Networks, Genetic algorithms and Formal Concept Analysis.

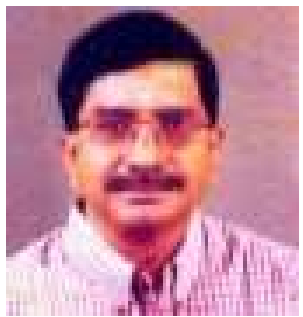

He has worked as Head, Chairman Board of Studies of Department of Informatics, Kakatiya University for 39 years, Chairman Board of Studies in Computer Science, SKD University, Ananthpur.Dean Faculty of Science, Kakatiya University.Presently working as Dean at Vaagdevi engineering College, Bollikunta, warangal since 2011 to present day. Guided many scholors from various universities and research areas are Computer Networking,, Cloud Computing, Network Communications, information Security and Machine learning. He has published many papers in national and international conferences and journals as author and coauthor. 